\title{
Christian Plantin: darle la palabra al conflicto
}

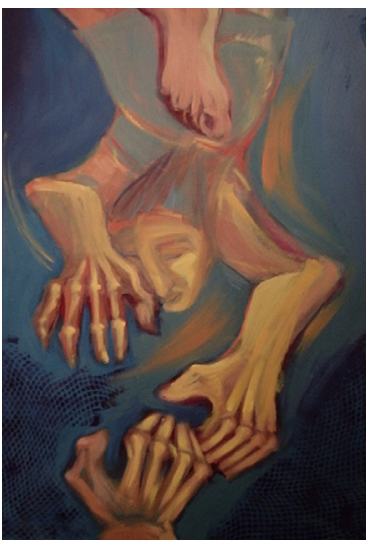

Alejandra Vitale (entrevista)

Universidad de Buenos Aires, Argentina

alejandravitale@filo.uba.ar

Laura Bonilla (transcripción)

Universidad de Buenos Aires/CONICET, Argentina

laura.bonilla369@gmail.com

Mariano Dagatti (edición)

Universidad de Buenos Aires/UNQ/CONICET, Argentina

onairamdagatti@gmail.com

Christian Plantin es Profesor Emérito de la Universidad de Lyon 2 (Francia) y ex director de investigación del Centre National de la Recherche Scientifique (CNRS). Trabaja en la Unidad de Investigación ICAR (Interacciones, Corpus, Aprendizajes, Representaciones). Sus trabajos versan sobre la argumentación, la interacción y la expresión lingüística de las emociones. Ha publicado, entre otros, L'argumentation (1995), L'argumentation - Histoire, théories, perspectives (2005), Les bonnes raisons des émotions (2013) -todos traducidos al español. Ha recibido la Medalla de Oro de la Comisión Francesa de la Cultura de Bruselas en 1989 y el "Distinguished Scholarship Award" de la Sociedad Internacional para el Estudio de la Argumentación (ISSA) en el 2001 ${ }^{1}$.

Esta entrevista fue realizada en el marco del IV Seminario Internacional de Discurso y Argumentación (SEDiar) que -organizado por el Instituto de Lingüística- se desarrolló en la Facultad de Filosofía y Letras de la UBA, entre el 14 y 16 de marzo de 2018. En este encuentro, el Prof. Plantin estuvo a cargo de la conferencia plenaria de cierre.

Alejandra Vitale: ¿Cómo sintetizaría su teoría dialogal de la argumentación? ¿Cuáles serían sus presupuestos y características?

Christian Plantin: En verdad, es muy sencillo. Me interesa construir un modelo -una representación formal- de la argumentación; es decir, ofrecer una representación de una actividad específica del lenguaje, que sea empíricamente adecuada y tenga capacidad explicativa. Mi teoría dialogal considera que la situación argumentativa típica se define por la existencia de una contradicción, es decir, de discursos antagonistas alrededor de una cuestión o de un problema.

1. Los conceptos mencionados aquí son definidos en: Plantin, C. Dictionnaire de l'argumentation, Lyon, ENS Éditions, 2016. Traducción y adaptación inglesa Dictionary of Argumentation, London, College Publications, 2018. 
Ahora bien, parto de la distinción de dos fenómenos amplios. Por un lado, tenemos la construcción habitual de una conversación o intercambio comunicativo, que presupone el acuerdo, la co-construcción y la coorientación del discurso entre las partes. Este es un hecho básico, bien reconocido, que ha sido tratado bajo diferentes nombres y con distintas designaciones. Por otro lado, tenemos la aparición y el tratamiento del desacuerdo, que es lo que realmente me interesa. ¿Qué sucede cuando se interrumpe esa co-construcción del discurso, esa dinámica colaborativa del diálogo?

Me permito echar luz sobre este problema argumentativo apelando a la noción médica de estasis o "congestión". Hay estasis cuando los humores están bloqueados; el arte médico trata de restablecer la circulación normal de los fluidos. Del mismo modo, hay una cuestión argumentativa cuando la circulación consensual del discurso está bloqueada por la aparición de una contradicción o de una duda; el arte de la argumentación intenta, en este sentido, restablecer el flujo normal, consensual, del diálogo. Los estados de estasis no aparecen, sin embargo, con una claridad meridiana. Por ejemplo, nosotros concertamos una cita para la entrevista, tuvimos dos o tres intercambios, yo digo 6:30, ustedes me dicen 6:20 y finalmente acordamos a las 6:25. Todo ocurre rápido, de manera muy eficaz, sin que pensemos demasiado en ello, sin que se vuelva memorable.

Desde mi punto de vista, sin embargo, la actividad argumentativa empieza con un desacuerdo ratificado; es decir, cuando no solo hay desacuerdo, sino que este es ratificado, sostenido y manifiesto. Entonces yo sé que ustedes saben que yo sé que no estamos de acuerdo y sé que las cosas no se van a resolver tan fácilmente porque hay diferencias que se mantienen más allá del intercambio inicial.

La actividad argumentativa no es una actividad de dialéctica, es más bien una actividad interaccional. Quiero decir, un modelo dialéctico supone dos personas que se ponen de acuerdo para resolver sus diferencias de opinión. Para mí, el juego argumentativo no se desarrolla entre dos personas sino entre por lo menos tres, es decir, que hay una instancia externa que ejerce un poder sobre nuestra diferencia, cuyo estatuto puede variar dependiendo de las circunstancias sociales. La sociedad no empieza con dos personas, sino con tres: la tercera persona encarnada, por ejemplo, por una institución, que introduce sus reglas argumentativas fundamentales e impone $u$ orienta el marco discursivo de la discusión.

¿Usted piensa que el analista de la argumentación tiene que explicitar su posición crítica frente a lo que analiza?, ¿habría una dimensión crítica del análisis argumentativo?, ¿qué postura adopta frente a esta cuestión?

Pienso que hay distintas posiciones. Conozco muy bien la tendencia de análisis del discurso que se define como una crítica social del discurso, el análisis del discurso de izquierda. Alguien me decía el otro día que era necesario adoptar una posición de izquierda para hacer análisis de discurso. Me parece un poco raro. Yo diría en cambio que la instancia evaluadora no es el analista. No veo al analista como el gran evaluador, como el encargado de dar por terminadas todas las disputas. Imaginemos que tenemos que evaluar una diferencia de opinión profunda que involucra intereses humanos. No corresponde pedirle al analista que nos diga quién habla de manera racional y quién está 
siendo irracional. Existen instituciones, especialistas, instancias de arbitrio, sistemas electorales, que bien pueden equivocarse, pero que tienen el derecho a rectificarse.

Desde mi punto de vista, el analista no es la vanguardia de la evaluación, la primera línea evaluativa. Ese es el primer punto. El segundo punto es qué pasa cuando el analista es a la vez profesor. Esa es otra historia. El profesor puede insuflarle aire al problema, mantenerlo vivo, puede hacer crecer y florecer la diferencia de opinión, pero a la vez tiene de alguna forma una responsabilidad social y científica, o sea que hay cosas que no se pueden decir porque simplemente son falsas y la universidad es una institución que está basada sobre la verdad. Mientras que el analista no es el evaluador supremo, el profesor de argumentación tiene tareas y responsabilidades que van de suyo con la deontología universitaria.

¿Ha seguido con interés las argumentaciones actuales en torno a los refugiados en la Unión Europea? ¿Tiene alguna posición al respecto? Sí, tomemos por ejemplo el caso de los refugiados, que es un tema muy vivo y muy interesante. Hace mucho tiempo, casi unos veinte años, me ocupé del tema de la nacionalidad francesa. Tuvimos todo un programa sobre este tema, habíamos organizado la discusión, nos habíamos provisto de documentación, información, habíamos realizado lecturas previas, y recuerdo muy bien la reacción de un estudiante, un varón adulto, de unos veinte o veinticinco años, que al finalizar la discusión dijo: "estamos discutiendo, pero no sabemos de qué estamos discutiendo". Su reflexión fue terrible.

La base de una buena argumentación es una documentación exhaustiva. Esa es la primera parte de mi respuesta. La segunda es que yo nunca trabajé como profesor ni como investigador sobre este tema. Está la figura del intelectual, claro, pero yo me defino más bien como un profesor investigador. La posición misma del intelectual dentro de la sociedad exige un largo debate, porque un intelectual es básicamente una persona que tiene una opinión fundada sobre ciertos asuntos debido a su erudición o expertise.

Sobre el tema de los refugiados, tengo una opinión, que es mi opinión como ciudadano y como ser humano. Considero que es, en primer lugar, un fenómeno grave, de una amplitud apenas sospechada y sobre la cual no se puede discutir livianamente. Es un fenómeno original, por otro lado; la inmigración actual no es la inmigración que venía de Argelia durante los sesenta para trabajar a buen precio en las fábricas francesas. Ahora se trata de otra cosa, se trata de guerras, de continentes enteros, del desorden climático, de regiones enteras destruidas por las guerras y el terrorismo. Es un hecho impresionante, sobre el cual no puedo decir mucho más. No es algo que vayamos a arreglar de forma inmediata, es una problemática que atravesará generaciones. Y del otro lado está Europa, con su bienestar, preocupada por su propia supervivencia, la de sus hijos y la de sus familias, cuyas angustias y miedos generan un terreno propicio para el renacimiento de una nueva forma de extrema derecha, como Marine Le Pen en Francia, una auténtica fascista, que afortunadamente fue derrotada en los últimos comicios.

¿Coincide con Perelman en que la argumentación es un antídoto contra la violencia? ¿Por qué no nos comenta su propia reflexión sobre la argumentación, la democracia y el pluralismo?

Quisiera decir dos cosas. En primer lugar, muchas veces, en Francia por lo menos, se olvidan las bases mismas de la actividad argumentativa. Suelo 
participar en reuniones en las que colegas profesores están muy interesados en los aspectos sociales de la argumentación, pero muy poco interesados en los cimientos, en el esqueleto mismo de la actividad argumentativa: reflexionar sobre la categorización, sobre la analogía, cuáles son los tipos de argumentos, cómo funciona el proceso argumentativo, cómo se vinculan las premisas con las conclusiones, la causalidad, por ejemplo. Yo pienso que es tiempo de darle más atención a la técnica de la argumentación, entendida como motor de la actividad argumentativa.

Ahora bien, ¿̇es la argumentación un antídoto contra la violencia, es un signo de la democracia? No estoy de acuerdo con esa idea, porque todas las dictaduras argumentan y argumentan siempre. La existencia de la argumentación como práctica no distingue una democracia de una dictadura. Son las instituciones. Quiero decir, se trata de reconocer si existen instituciones que te permiten hablar y que te permiten terminar una discusión, salir a la calle y seguir vivo. No es la argumentación la que te garantiza eso, son las instituciones de un país libre; el ejercicio democrático de la argumentación presupone y fortalece la democracia, pero en las dictaduras no se deja de argumentar.

Hace mucho, en 2006, yo estaba acá en Buenos Aires y estaba leyendo todos los carteles que se ponen en su Universidad, en Puán, cuando vi una frase que me pareció maravillosa: "La argumentación da palabra al conflicto". Dar palabra al conflicto, ese sería el papel fundamental. Si hay algo que he aprendido en América latina, desde México hasta Río Gallegos, es la importancia de abandonar una visión de la argumentación como intercambio dialéctico, como si se tratara de la esfera pública de Habermas. El espacio argumentativo es un espacio socialmente estructurado, donde se juegan las disputas de poder, de violencia legal e ilegal, desde la policía hasta el narcotráfico. Por eso pienso que la argumentación, las técnicas argumentativas, pueden hacer mucho, dado que estamos protegidos por una organización republicana y democrática que nos garantiza los espacios para la argumentación. Y la característica de un verdadero espacio argumentativo en democracia es que puedes discutir y que puedes salir a la calle y sobrevivir.

\section{¿Cuáles son sus proyectos actuales?}

Acaba de aparecer la versión inglesa de mi Diccionario de la Argumentación, cuya traducción y adaptación me tomó muchísimo tiempo. Y lo que estoy haciendo ahora es retomar un proyecto sobre las emociones en el discurso. Ustedes conocen mi libro Las buenas razones de las emociones, que fue traducido al español por la Universidad de Moreno. Ahora trato de retomar este tema de la argumentación por las emociones con una orientación más franca hacia el análisis del discurso: me refiero a un desarrollo conceptual y al diseño de principios y métodos de análisis, que tengan en cuenta las dinámicas sociales e interindividuales. Estoy escribiendo una contribución sobre Emotions in affects para una Enciclopedia de la Interacción Social. Me interesa desarrollar un estudio de la emoción como actuación o interacción en circunstancias disruptivas positivas o negativas con variación de intensidad, cómo todo eso se construye dentro del discurso, dentro de un discurso ordinario y también dentro de uno muy elaborado.

Muchísimas gracias, no solo por sus ideas sino por haber hablado en español. 